\title{
ESPÉCIES INDICADORAS DE RESÍDUOS DE SAFLUFENACIL EM SOLOS
}

\author{
INDICATORS SPECIES OF SAFLUFENACIL RESIDUE IN SOILS
}

Lucas Heringer Barcellos Júnior ${ }^{a}$, Luciane Renata Agazzi ${ }^{a}$, Gustavo Antonio Mendes Pereira ${ }^{a}$, Elisa Maria Gomes da Silva ${ }^{a}$, Alba Rocio Aguilar Piratoba ${ }^{a}$, Paulo Sérgio Ribeiro de Souza ${ }^{\mathrm{a}}$, Antonio Alberto da Silva

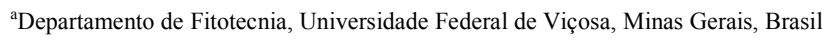

*Autor correspondente: lucasheringerbj@hotmail.com.

\section{INFORMAÇÕES DO ARTIGO}

Histórico do artigo:

Recebido: 22 Janeiro 2019.

Aceito: 15 Julho 2019.

Publicado: 25 Agosto 2019

\section{Palavras-chave/Keywords:}

Comportamento no Solo/ Behavior in

Soil.

Ensaio Biológico/Bioassay.

Herbicida/ Herbicide.

\section{Financiamento:}

O presente trabalho foi realizado com apoio da Coordenação de Aperfeiçoamento de Pessoal de Nível Superior - Brasil (CAPES) - Código de Financiamento 001, do Conselho Nacional de Desenvolvimento Científico e Tecnológico (CNPq) e Fundação de Amparo à Pesquisa do Estado de Minas Gerais (FAPEMIG). Os autores agradecem pelas bolsas de pesquisa.

Direito Autoral: Este é um artigo de acesso aberto distribuído sob os termos da Licença Creative Commons, que permite uso, distribuição e reprodução irrestritos em qualquer meio, desde que $\mathrm{o}$ autor e a fonte originais sejam creditados.

\section{Citação deste artigo:}

BARCELLOS JÚNIOR, L. H.; AGAZZI, L. R.; PEREIRA, G. A. M.; DA SILVA, E. M. G.; PIRATOBA, A. R. A.; SOUZA, P. S. R.; SILVA, A. A. Espécies indicadoras de resíduos de saflufenacil em solos. Revista Brasileira de Herbicidas, v. 18, n. 2. 2019.

\begin{abstract}
RESUMO
Um dos métodos, eficientes e de baixo custo, para estimar a presença de herbicidas no solo é o biológico. Entretanto, antes de se utilizar este método é necessário identificar espécies que apresentam alta sensibilidade ao herbicida em estudo. Esta pesquisa teve como objetivo selecionar espécies que apresentem alta sensibilidade ao saflufenacil para posteriores estudos da dinâmica desse herbicida no solo. Foram avaliadas a sensibilidade de sete espécies de plantas ao saflufenacil (Curcubita pepo, Beta vulgaris, Daucus carota, Citrullus lanatus, Cucumis sativus, Sorghum bicolor e Solanum lycopersicum). Para isso, estas espécies foram cultivadas em substrato inerte (areia lavada) tratado com doses crescentes do saflufenacil $\left(0,0 ; 0,625 ; 1,25 ; 2,50 ; 5,0 ; 10,0\right.$ e $\left.20,0 \mathrm{~g} \mathrm{ha}^{-1}\right)$. As espécies $B$. vulgaris, $S$. lycopersicum e $S$. bicolor foram as mais sensíveis ao saflufenacil. Dentre estas espécies, $B$. vulgaris é de fácil cultivo, crescimento rápido e permite rápida visualização dos sintomas de intoxicação. Concluiu-se que $B$. vulgaris pode ser considerada como indicadora da presença do saflufenacil na solução do solo para estudos da dinâmica desse herbicida em solos.
\end{abstract}

\begin{abstract}
One of the methods, efficient and low cost, to estimate the presence of herbicides in the soil is the biological. However, before using this method it is necessary to identify species that have high sensitivity to the herbicide under controlled conditions. This research aimed to select species that show high sensitivity to saflufenacil for later studies of the dynamics of this herbicide in soil. The sensitivity of seven plants species to saflufenacil (Curcubita pepo, Beta vulgaris, Daucus carota, Citrullus lanatus, Cucumis sativus, Sorghum bicolor and Solanum lycopersicum) were evaluated. For this, these species were cultivated on an inert substrate (washed sand) treated with increasing doses of saflufenacil $(0.0,0.625,1.25,2.50$, 5.0, 10.0 and $20.0 \mathrm{~g} \mathrm{ha}^{-1}$ ). The species B. vulgaris, S. lycopersicum and S. bicolor were the most sensitive to saflufenacil. Among these species, B. vulgaris is easy to grow, fast growing and allows rapid visualization of the symptoms of intoxication. It was concluded that $B$. vulgaris can be considered as an indicator of the presence of saflufenacil in the soil solution to study the dynamics of this herbicide in soils.
\end{abstract}




\section{Introdução}

Os herbicidas são os agrotóxicos mais consumidos no mundo, com destaque no Brasil que é hoje o maior consumidor, sendo responsável pelo uso de mais de $220 \mathrm{mil}$ toneladas de ingrediente ativo ao ano (FAO, 2018). Apesar desta grande demanda por herbicidas, o que se observa no país é que muitas das vezes estes produtos têm sido utilizados de maneira incorreta. Em razão disso é comum relatos de sérios problemas de contaminações dos ecossistemas. Isto coloca em risco a segurança da vida e o uso da água (VÖRÖSMARTY et al., 2010; BEKETOV et al., 2013; ZHANG et al., 2013; VAN STEMPVOORT et al., 2014; TOPAZ et al., 2018).

Independente de um herbicida ser aplicado em pré ou em pós-emergência, a grande maioria de suas moléculas tem como destino final o solo (LAW, 2001). Ao atingir o solo, o herbicida está sujeito a processos que influenciam seu destino no ambiente (ALEKSEEVA et al., 2014). Em razão disso, o conhecimento da dinâmica dos herbicidas no ambiente é imprescindível para que os profissionais possam utilizar o método químico de controle das plantas daninhas garantindo a eficiência do tratamento e reduzindo os riscos de contaminação ambiental.

Para se compreender a dinâmica do herbicida no ambiente são necessários diversos estudos referentes a sorção, a lixiviação e a persistência de suas moléculas nos diferentes tipos de solo. Conhecendo-se estas variáveis, é possível fazer uma estimativa do possível destino dos herbicidas no ambiente (SOUZA et al., 2016; KERMINEN et al., 2018; MENDES et al., 2018). Os estudos mencionados acima podem ser realizados por métodos biológico e químico ou pela associação dos dois métodos (ANDRADE et al., 2010; BRAGA et al., 2016; FARIA et al., 2018).

A quantificação da sorção de um herbicida no solo normalmente é realizada por cromatografia (MENDES et al., 2016). Contudo, diversas pesquisas têm evidenciado a possibilidade de estimar a sorção utilizando-se testes biológicos (ANDRADE et al., 2010, BRAGA et al., 2016, FARIA et al., 2018). Nos ensaios biológicos é realizada a monitoração ativa, pois neles são utilizados organismosteste, altamente sensíveis ao herbicida. Normalmente são realizados em ambiente protegido que permite ótimo crescimento da espécie indicadora (RAYA-RODRIGUEZ, 2000). Sua utilização fundamenta-se na exposição dos organismos-teste a várias concentrações de uma ou mais substâncias, ou fatores ambientais, durante um período de tempo suficiente para ocorrer à resposta biológica (GHERARDI-GOLDSTEIN et al., 1990).

O primeiro passo para a realização de um bioensaio envolvendo herbicidas é a seleção de plantas que apresentem alta sensibilidade a um composto específico e seja de fácil condução em substratos em casa de vegetação, além é claro, de apresentarem sintomatologia de fácil visualização para a realização de avaliações de intoxicação.

Para muitos herbicidas já existem resultados de pesquisa que indicam quais plantas podem ser utilizadas como indicadoras para detecção de seus resíduos no ambiente. Entretanto, as moléculas que estão sendo incorporadas recentemente no mercado carecem desse tipo de estudo, como é o caso do saflufenacil \{2-chloro-4-fluoro5-[3-methyl-2,6-dioxo-4-(trifluoromethyl) pyrimidin-1-yl]$\mathrm{N}$-[methyl (propano-2-yl) sulfamoylbenzamide\}. Este herbicida está sendo muito utilizado no Brasil em préemergência para o controle de plantas daninhas eudicotiledôneas nas principais culturas agrícolas do país (MAPA, 2018). Além disso é uma alternativa muito utilizada no manejo de plantas daninhas resistentes a outros herbicidas como a buva (Conyza sp.) (BUDD et al., 2017; MONTGOMERY et al., 2017) e o caruru palmeri (Amaranthus palmeri) (CROW et al., 2015; RIOS et al., 2016).

Diversos autores (VIVIAN et al., 2006; MELO et al., 2010; FREITAS et al., 2014; BRAGA et al., 2016) relataram que o sorgo (Sorghum bicolor) pode ser utilizado como espécie indicadora da presença de herbicidas inibidores da PROTOX na solução do solo. Entretanto, em nenhum desses estudos foi avaliado o saflufenacil. Já estudos realizados por Gannon et al. (2014) indicam a canola (Brassica napus) como espécie altamente sensível ao saflufenacil. Este ensaio foi realizado nos Estados Unidos, em condições climáticas muito diferentes das encontradas no Brasil. É importante salientar que para uma espécie ser considerada ideal para ensaios biológicos ela deve ser de fácil cultivo, crescimento rápido e permitir rápida visualização dos sintomas de intoxicação.

Esta pesquisa teve por objetivo selecionar uma espécie vegetal para ser utilizada como indicadora para estudos da dinâmica do saflufenacil em solos tropicais.

\section{Material e métodos}

Este ensaio foi conduzido em casa de vegetação em condições controladas de temperatura e irrigação, na Universidade Federal de Viçosa. O primeiro passo foi selecionar quais espécies seriam testadas, onde o critério adotado para seleção foram plantas que apresentavam histórico de sensibilidade a herbicidas no campo, que apresentam rápido crescimento e que se adaptam bem às condições de vasos e casa de vegetação. Com isto as seguintes espécies foram escolhidas: abóbora (Curcubita pepo), beterraba (Beta vulgaris), cenoura (Daucus carota L.), melancia (Citrullus lanatus), pepino (Cucumis sativus L.), sorgo (Sorghum bicolor) e tomate (Solanum lycopersicum L.).

Todas essas espécies vegetais foram semeadas em substrato inerte (areia lavada). Este substrato foi peneirado em malha de $4 \mathrm{~mm}$ e lavado em água corrente até retirar o excesso de impurezas. Posteriormente, a areia ficou incubada com ácido clorídrico PA, diluído em água, numa concentração de $600 \mathrm{~mL}$ de ácido para cada $10 \mathrm{~L}$ de água. Manteve-se por 36 horas uma lâmina de $10 \mathrm{~cm}$ da solução ácida acima do nível da areia, para queima do material orgânico presente. Após esse período, novamente a areia foi lavada em água corrente para retirar o excesso de ácido, elevando-se o $\mathrm{pH}$ próximo a 7,0.

$\mathrm{O}$ ensaio foi conduzido em delineamento inteiramente casualizado, em esquema fatorial $7 \times 7$, com quatro repetições, em que o primeiro fator constituiu-se das 
sete espécies testadas, e o segundo fator, de doses crescentes do herbicida $\left(0 ; 0,625 ; 1,25 ; 2,50 ; 5,0 ; 10,0\right.$ e $20,0 \mathrm{~g} \mathrm{ha}^{-1}$ de saflufenacil (Heat, $700 \mathrm{~g} \mathrm{~kg}^{-1}$ i. a., WG, BASF).

As unidades experimentais foram constituídas por vasos plásticos de polipropileno com capacidade para 0,3 $\mathrm{dm}^{3}$. Para a aplicação do herbicida na superfície dos vasos utilizou-se o pulverizador de precisão, pressurizado a $\mathrm{CO}_{2}$, equipado com barra com duas pontas TT 11002, espaçadas de $0,50 \mathrm{~m}$, mantidos à pressão de $25 \mathrm{lb} \mathrm{pol}^{-2}$ e volume de calda de $150 \mathrm{~L} \mathrm{ha}^{-1}$. Logo após a aplicação, o substrato foi homogeneizado e realizou-se a semeadura das espécies. Durante a condução do experimento, os vasos foram irrigados diariamente, sendo mantidos próximos à capacidade de campo e, para o melhor desenvolvimento das plantas, estas receberam solução nutritiva completa, seguindo recomendações de Hoagland e Arnon (1950).

Aos 21 dias após a emergência das plantas (DAE) foi avaliado a percentagem de intoxicação atribuindo notas de 0 (ausência de sintoma) a 100 (morte da planta) (ALAM, 1974).

Aos 21 DAE realizou-se a colheita do experimento, que consistiu no corte da parte aérea na região do coleto das plantas e retirada das raízes em água corrente. Posteriormente, esse material foi acondicionado em uma estufa de circulação forçada de ar $\left(70 \pm 2{ }^{\circ} \mathrm{C}\right)$ até atingir

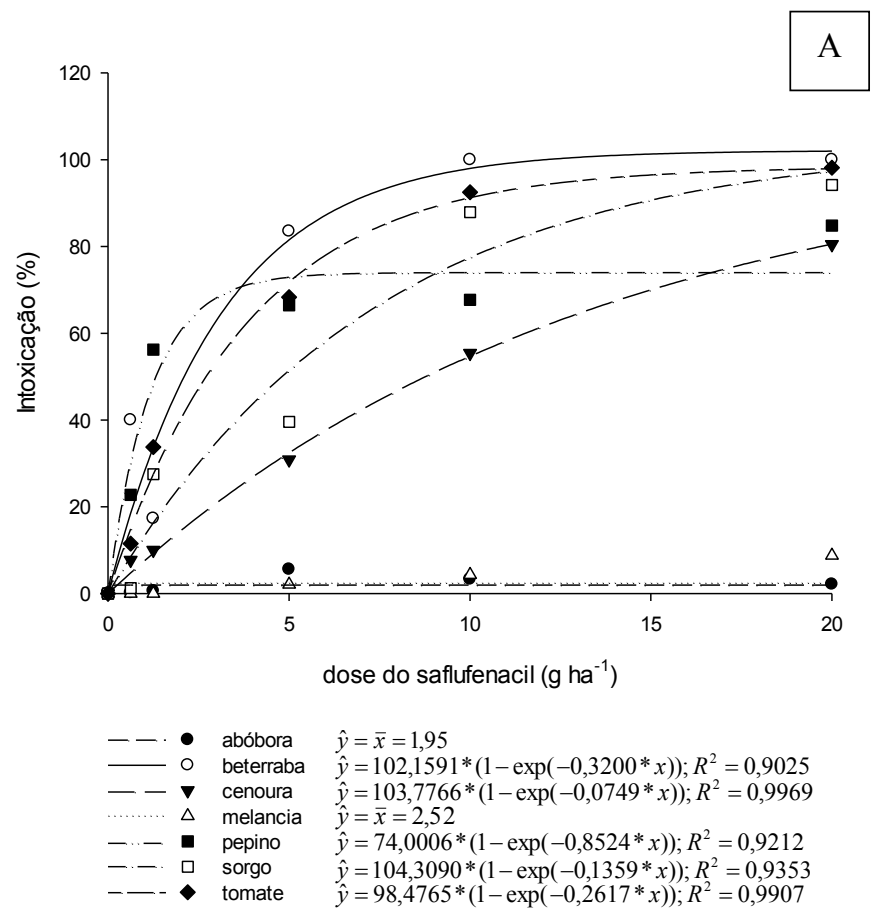

massa constante, em seguida foi mensurada a massa de matéria seca da parte aérea, raiz e total da planta em uma balança de precisão de $0,0001 \mathrm{~g}$. A massa de matéria seca foi expressa em percentagem em relação à testemunha.

Os dados obtidos foram submetidos a modelos exponenciais de dose-resposta do herbicida. Os modelos foram escolhidos para cada espécie em função do comportamento biológico. Os valores de $\mathrm{C}_{50}$ foram obtidos por meio das equações ajustadas do modelo de regressão para cada espécie, correspondendo à dose necessária em causar intoxicação ou redução de $50 \%$ no crescimento das plantas testadas.

\section{Resultados e discussão}

Dentre as espécies estudadas, a beterraba, o tomate e o sorgo foram as espécies que mais se mostraram sensíveis à presença do saflufenacil (Figura 1A). A maior dose testada (20 $\mathrm{g} \mathrm{ha}^{-1}$, referente a $20 \%$ da dose recomendada) resultou em morte destas espécies. O pepino e a cenoura apresentam tendência de aumento de intoxicação com o aumento da dose. No entanto, essas espécies tiveram valores máximos de intoxicação em torno de $80 \%$. Já a abóbora e a melancia não se mostraram sensíveis ao saflufenacil, apresentando intoxicação inferior à $5 \%$.

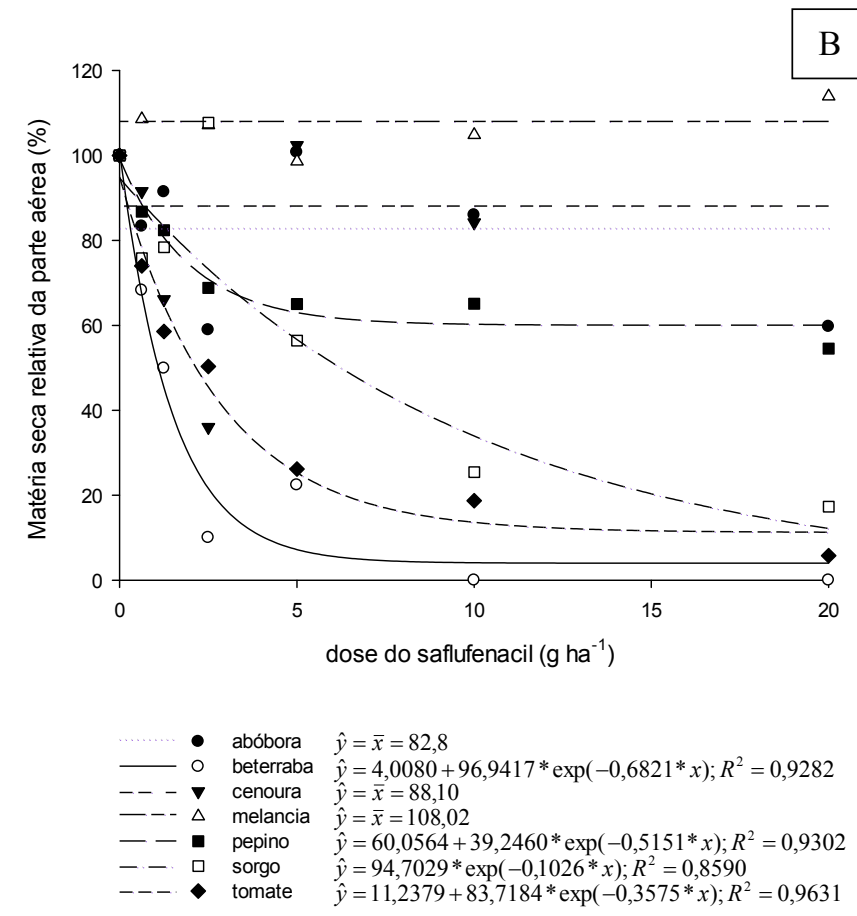

Figura 1. Porcentagem de intoxicação (A) e de matéria seca relativa da parte aérea (B) de plantas de abóbora, beterraba, cenoura, melancia, pepino, sorgo e tomate, cultivadas em vaso após a aplicação de diferentes doses do saflufenacil em areia, avaliado aos 21 DAE.

As variáveis matéria seca da parte aérea, matéria seca do sistema radicular e matéria seca total apresentaram comportamento semelhante quanto à sensibilidade das espécies em estudo ao saflufenacil (Figuras 1B, 2A e 2B). De forma geral, houve redução da matéria seca da parte aérea da beterraba, do tomate, do sorgo e pepino. Apesar da tendência de redução da matéria seca da parte aérea do pepino em doses crescentes do herbicida, as plantas apresentaram mais de $60 \%$ da matéria seca quando comparado com a testemunha sem herbicida. A abóbora, cenoura e melancia não tiveram redução da matéria seca da parte aérea (Figura 1B). 
L. H. BARCELLOS JÚNIOR et al.
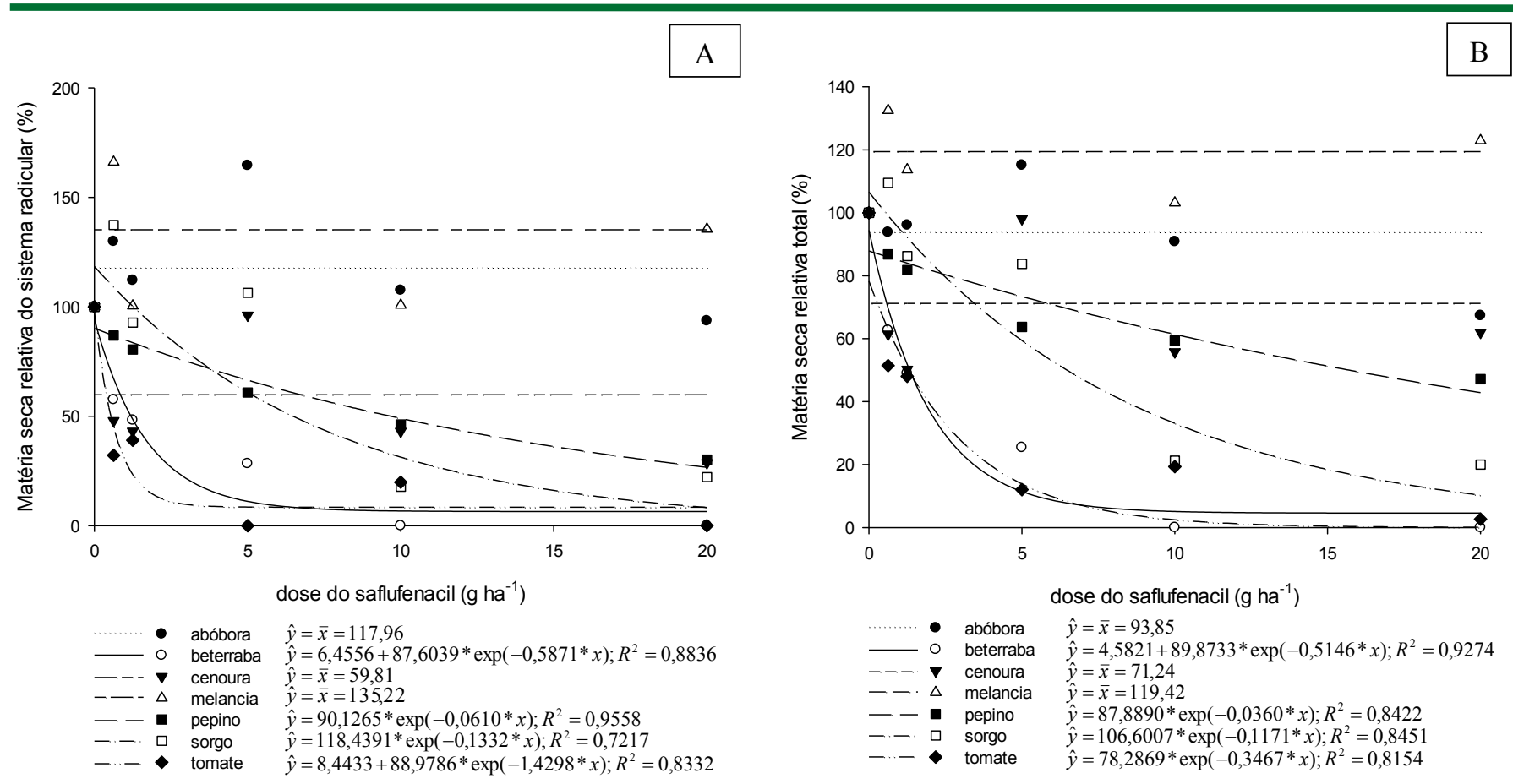

Figura 2. Porcentagem de matéria seca relativa do sistema radicular (A) e de matéria seca relativa total (B) de plantas de abóbora, beterraba, cenoura, melancia, pepino, sorgo e tomate, cultivadas em vaso após a aplicação de diferentes doses do saflufenacil em areia, avaliado aos 21 DAE.

A variável percentagem de intoxicação mostrou ser melhor indicador para avaliar a sensibilidade das espécies testadas ao saflufenacil. Para o sulfentrazone, herbicida inibidor do mesmo mecanismo de ação do saflufenacil, a matéria seca da raiz e parte aérea mostraram-se pouco eficientes para avaliar a sensibilidade ao herbicida (SZMIGIELSKI et al., 2009). A identificação dos sintomas visuais nas plantas é mais perceptível, para este herbicida, devido a inibição da protoporfirinogênio oxidase IX, enzima chave para a produção da clorofila. Com o acúmulo do protoporfirinogênio IX no cloroplasto e seu posterior deslocamento para o citoplasma das células, ocorrem as necroses nos tecidos foliares provocadas pela peroxidação dos lipídios das membranas celulares em presença de luz (WELLER, 2003).

Robinson e McNaughton (2012) avaliando injúrias causadas por carryover de saflufenacil, em espécies semeadas um ano após a aplicação do herbicida, observaram que o pepino e a beterraba tiveram redução da matéria seca em doses mais baixas quando comparado com a cenoura. A beterraba apresentou elevados sintomas de intoxicação e maiores reduções de matéria seca quando tratadas com mesotrione (RIDDLE et al., 2013), herbicida de outro mecanismo de ação, mas que também afeta os processos fotossintéticos da planta. Esses resultados corroboram com os encontrados neste trabalho, evidenciando maior sensibilidade da beterraba ao saflufenacil.

A (Tabela 1) expressa os valores da dose necessária para causar $50 \%$ de intoxicação e redução da matéria seca nas espécies que demonstraram ser potencialmente sensíveis ao saflufenacil. A beterraba foi a espécie que apresentou menor valor de $C_{50}$ para as variáveis em estudo, com exceção do sintoma de intoxicação e matéria seca do sistema radicular, onde o pepino e o tomate foram as espécies com menor $\mathrm{C}_{50}$, respectivamente. Apesar de o pepino ter sido a espécie que exigiu menor dose para causar $50 \%$ de intoxicação, isso não pode ser atribuído para apontar esta espécie como a mais sensível ao saflufenacil, pois a maior dose em estudo não foi suficiente para promover a morte da planta. Os valores máximos de intoxicação para esta espécie foram em torno de $80 \%$ (Figura 1A). O saflufenacil não causou grandes reduções na matéria seca da parte aérea e matéria seca total do pepino, o que impossibilitou a estimativa da $C_{50}$ para essa espécie nestas variáveis (Tabela 1). 
Tabela 1. Dose necessária em proporcionar $50 \%$ de resposta $\left(\mathrm{C}_{50}\right)$ para as variáveis intoxicação, matéria seca da parte aérea (MSPA), matéria seca do sistema radicular (MSSR) e matéria seca total (MST).

\begin{tabular}{|c|c|c|c|c|}
\hline \multirow[t]{2}{*}{ Espécies } & \multicolumn{4}{|c|}{$\begin{array}{c}\mathrm{C}_{50}{ }^{/ 1} \\
\left(\mathrm{~g} \mathrm{ha}^{-1}\right)\end{array}$} \\
\hline & Intoxicação & MSPA & MSSR & MST \\
\hline Beterraba & 2,1 & 1,1 & 1,2 & 1,3 \\
\hline Pepino & 1,4 & $\mathrm{ND}^{12}$ & 9,7 & $\mathrm{ND}^{/ 2}$ \\
\hline Sorgo & 4,8 & 6,2 & 6,5 & 6,5 \\
\hline Tomate & 2,7 & 2,2 & 0,6 & 1,3 \\
\hline
\end{tabular}

${ }^{/ 1}$ Dose necessária para proporcionar $50 \%$ de intoxicação ou redução de $50 \%$ na matéria seca; ${ }^{12}$ Não detectado.

Riddle et al. (2013) observaram intoxicação severa da beterraba, causando a morte dessa cultura, enquanto o pepino nas maiores doses do mesotrione apresentou apenas $55 \%$ de intoxicação. Assim como observado neste trabalho, esses autores também obtiveram menores valores de $\mathrm{C}_{50}$ para a beterraba. Entre as espécies que se mostraram promissoras em ser sensíveis a este herbicida, a beterraba foi a que apresentou sintomas de intoxicação e redução do crescimento em $50 \%$ com as menores doses do herbicida (Tabela 1).

O tomate foi uma espécie que apresentou sensibilidade ao saflufenacil semelhante à beterraba para quase todas as variáveis (Tabela 1). Para a matéria seca do sistema radicular apresentou $\mathrm{C}_{50}$ menor do que as outras espécies, demonstrando ser altamente sensível ao herbicida. Todavia, devido ao mecanismo de ação do saflufenacil, a matéria seca do sistema radicular não é tão expressiva (SZMIGIELSKI et al., 2009). Observando os valores de $\mathrm{C}_{50}$ para os sintomas de intoxicação e matéria seca da parte aérea (Tabela 1), percebe-se maior susceptibilidade da beterraba.

A alta sensibilidade da beterraba pode ser explicada devido a redução da enzima glutationa S-transferase quando essa espécie é submetida ao herbicida. Plantas de beterraba sensíveis ao chlorymuron-ethyl apresentaram redução significativa da atividade dessa enzima quando comparado com beterrabas tolerantes (WEI et al., 2010). A glutationa Stransferase no citosol das células ativa o tripeptídeo glutationa, responsáveis pela conjugação de substratos tóxicos como herbicidas, inativando o produto químico (EDWARDS et al., 2000). É provável que a beterraba apresente altos níveis de glutationa S-transferase. Contudo, quando tratadas com herbicidas, a concentração dessa enzima diminui tornando-a muito suscetível ao composto químico.

\section{Conclusão}

A beterraba (Beta vulgaris) é a espécie mais indicada para a condução de ensaios biológicos com o fim de detectar a presença do saflufenacil em solo nas condições brasileiras.

\section{Referências}

ALAM - Asociación Latinoamericana De Malezas. Recomendaciones sobre unificación de los sistemas de evaluación en ensayos de control de malezas. ALAM, v.1, n.1, p.35-38, 1974.

Alekseeva, T.; Kolyagin, Y.; Sancelme, M.; Besse-Hoggan, P. Effect of soil properties on pure and formulated mesotrione adsorption onto vertisol (Limagne plane, Puy-de -Dome, France). Chemosphere, v. 111, p. 177-183, 2014.

Andrade, S. R. B.; Silva, A. A.; Lima, C. F.; D’antonino, L.; Queiroz, M. E. L. R.; França, A. C.; Felipe, R. S.; Victoria Filho, R. Lixiviação do ametryn em Argissolo VermelhoAmarelo e Latossolo Vermelho-Amarelo, com diferentes valores de pH. Planta Daninha, v. 28, n. 3, p. 655-663, 2010 .

Beketov, M. A.; Kefford, B. J.; Schäfer, R .B.; Liess, M. Pesticides reduce regional biodiversity of stream invertebrates. Proceedings of the National Academy of Sciences of United States of America, v. 110, p. 1103911043, 2013.

Braga, D. F.; Freitas, F. C. L.; Rocha, P. R. R.; Araújo, A. G. D.; Melo, V. C. Leaching of Sulfentrazone in Soils from the Sugarcane Region in the Northeast Region of Brazil. Planta daninha, v.34, n.1, p. 161-169, 2016.

Budd, C. M.; Soltani, N.; Robinson, D. E.; Hooker, D. C.; Miller, R. T.; Sikkema, P. H. Efficacy of Saflufenacil for Control of Glyphosate-Resistant Horseweed (Conyza canadensis) as Affected by Height, Density, and Time of Day. Weed Science, v. 65, n. 2, p. 275-284, 2017.

Crow, W. D.; Steckel, L. E.; Hayes, R. M.; Mueller, T. C. Evaluation of Post-Harvest Herbicide Applications for Seed Prevention of Glyphosate-Resistant Palmer amaranth (Amaranthus palmeri). Weed technology, v. 29, ed. 3, p. 405-411, 2015.

Edwards, R.; Dixon, D. P.; Walbot, V. Plant glutathione Stransferases: enzymes with multiple functionsin sickness and in health. Trends in Plant Science, v. 5, n. 5, p. 193198, 2000. 
FAO (2018) - Food and Agriculture Organization of the United Nations. FAOSTAT. Disponível em: <http:// www.fao.org/faostat/en/\#data/RP $>$. Visualizado em: 16 de março de 2018 .

Faria, A. T.; Souza, M. F.; Passos, A. B. R. J.; Silva, A. A.; Silva, D. V.; Zanuncio, J. C.; Rocha, P. R. R. Tebuthiuron leaching in three Brazilian soils as affected by soil $\mathrm{pH}$. Environmental Earth Sciences, v. 77, p. 1-12, 2018.

Freitas, M.A.M.; Passos, A.B.R.J.; Torres, L.G.; Moraes, H.M.F.; Faustino, L.A.; Rocha, P.R.R.; Silva, A.A. Sorção do sulfentrazone em diferentes tipos de solo determinada por bioensaios. Planta Daninha, Viçosa-MG, v. 32, n. 2, p. 385 $-392,2014$.

Gannon, T. W.; Hixson, A. C.; Keller, K. E.; Weber, J. B.; Knezevic, S. Z.; Yelverton, F. H. Soil Properties Influence Saflufenacil Phytotoxicity. Weed Science, v. 62, n. 657$663,2014$.

Gherardi-Goldstein, E.; Bertoletti, E.; Zagatto, P. A.; Araújo, R. P. A.; Ramos, M. L. L. C. Procedimentos para Utilização de Testes de Toxicidade no Controle de Efluentes Líquidos, Companhia de Tecnologia de Saneamento Ambiental (CETESB): São Paulo, 1990.

Hoagland, D.; Arnon, D. I. The water culture method for growing plants without soil. California Agriculture Experimental Station Circular, 1950. 347 p.

Kerminen, K.; Le Moel, R.; Harju, V.; Kontro, M. H. Influence of organic matter, nutrients, and cyclodextrin on microbial and chemical herbicide and degradate dissipation in subsurface sediment slurries. Science of the Total Environment, v. 618, p. 1449-1458, 2018.

Law, S. E. Agricultural electrostatic spray application: a review of significant research and development during de $20^{\text {th }}$ century. Journal Electrostatic, v. 51-52, p. 25-42, 2001 .

MAPA - Ministério da Agricultura, Pecuária e Abastecimento. Disponível em: < http:// agrofit.agricultura.gov.br/agrofit_cons/!

ap_produto_form_detalhe_cons?

p_id_produto_formulado_tecnico $=9299 \& p \_t i p o \_j a n e l a=N E$ $\mathrm{W}>$. Acesso em: 15 mar. 2018 .

Melo, C.A.D.; Medeiros, W.N.; Tuffi Santos, L.D.; Ferreira, F.A.; Tiburcio, R.A.S.; Ferreira, L.R. Lixiviação de sulfentrazone, isoxaflutole e oxyfluorfen no perfil de três solos. Planta Daninha, v. 28, n. 2, p. 385-392, 2010.

Mendes, K. F.; Reis, M. R.; Passos, A. B. R. J.; Inoue, M. H.; Silva, A. A.; Silva, D. V. Determination of oxadiazon residues in the field treated soil with and without organic matter incorporated. Environmental Earth Sciences, v. 75, n. 222 , p. 1-8, 2016.
Mendes, K.F.; Hall, K.E.; Takeshita, V.; Rossi, M. L.; Tornisielo, V. L. Animal bonechar increases sorption and decreases leaching potential of aminocyclopyrachlor and mesotrione in a tropical soil. Geoderma, v. 316, p. 11-18, 2018.

Montgomery, G. B.; Treadway, J. A.; Reeves, J. L.; Steckel, L. E. Effect of Time of Day of Application of 2,4-D, Dicamba, Glufosinate, Paraquat, and Saflufenacil on Horseweed (Conyza canadensis) Control. Weed Technology, v. 31, n. 4, p. 550-556, 2017.

Raya-Rodriguez, M. T. O Uso de bioindicadores para Avaliação da Qualidade do Ar em Porto Alegre. In: Zurita, M. L. L.; Tolfo, A.M. (Org.) A Qualidade do Ar em Porto Alegre. Porto Alegre, Secretaria Municipal do Meio Ambiente. 2000. p.68-76.

Riddle, R. N.; O'Sullivan, J.; Swanton, C. J.; Acker, R. C. $\mathrm{V}$. Field and Greenhouse Bioassays to Determine Mesotrione Residues in Soil. Weed Technology, v. 27, n. 3, p. 565-572, 2013.

Rios, S. I.; Wright, S. D.; Banuelos, G.; Shrestha, A. Tolerance of Amaranthus palmeri populations from California to postemergence herbicides at various growth stages. Crop protection, v. 87, p. 6-12, 2016.

Robinson, D. E.; McNaughton, K. E. Saflufenacil Carryover Injury Varies among Rotational Crops. Weed Technology, v. 26, n. 2, p. 177-182, 2012.

Souza, M.F., Neto, M.D.C., Marinho, M.I., Saraiva, D.T., Faria, A.T., Silva, A.A., Silva, D.V. Persistence of imidazolinones in soils under a clearfield system of rice cultivation. Planta Daninha, v. 34, n. 3, p. 589-596, 2016.

Szmigielski, A. M.; Schoenau, J. J.; Johnson, E. N.; Holm, F. A.; Sapsford, K. L.; Liu, J. Development of a Laboratory Bioassay and Effect of Soil Properties on Sulfentrazone Phytotoxicity in Soil. Weed Technology, v. 23, n. 3, p. 486491, 2009.

Topaz, T., Egozi, R., Eshel, G., Chefetz, B. Pesticide load dynamics during stormwater flow events in Mediterranean coastal streams: Alexander stream case study. Science of the Total Environment, v. 625, p. 168-177, 2018.

Vivian, R.; Reis, M. R.; Jakelaitis, A.; Silva, A. F.; Guimarães, A. A.; Santos, J. B.; Silva, A. A. Persistência de sulfentrazone em Argissolo Vermelho-Amarelo cultivado com cana-de-açúcar. Planta Daninha, v. 24, n. 4, p. 741750,2006

Van Stempvoort, D. R.; Roy, J. W.; Brown, S. J.; Bickerton, G. Residues of the herbicide glyphosate in riparian groundwater in urban catchments. Chemosphere, v. 95, p. 455-463, 2014.

Vörösmarty, C. J.; Mcintyre, P. B.; Gessner, M. O.; 
Dudgeon, D.; Prusevich, A.; Green, P.; Glidden, S.; Bunn, S. E.; Sullivan, C. A.; Liermann, C. R.; Davies, P. M. Global threats to human water security and river biodiversity. Nature, v. 467, p. 555-561, 2010.

Wei, D.; Feng-ming, M. A.; Zhuo, C.; Bo, T. Effect of Chlorimuron-Ethyl on Biochemical Mechanism in Tolerant Sugar Beet. Agricultural Sciences in China, v. 9, n. 12, p. 1771-1776, 2010.

Weller, S. Diquat, paraquat, diphenylethers and oxadiazon uses and mechanism- of action. In: Herbicide action course. West Lafayette: Purdue University, 2003. p.185224.

Zhang, C.; Liao, X.; Li, J.; Xu, L.; Liu, M.; Du, B.; Wang Y. Influence of long-term sewage irrigation on the distribution of organochlorine pesticides in soil-groundwater systems. Chemosphere, v. 92, p. 337-343, 2013. 